\title{
AVANÇOS NO INTERCÂMBIO DE DADOS DO PROJETO DE INSTALAÇÕES: DO CAD 3D AO BIM EM EXPERIÊNCIAS REPRESENTATIVAS ${ }^{1}$
}

\author{
ADVANCES IN DATA EXCHANGE OF MEP DESIGN: FROM CAD3D TO BIM \\ IN REPRESENTATIVE EXPERIMENTS
}

\author{
Lidiane Maria Batista \\ Instituto de Pesquisas Tecnológicas (IPT) \\ lidiane.mbatista@gmail.com \\ Sérgio Leal Ferreira \\ Universidade de São Paulo (USP) \\ sergio.leal@usp.br
}

\begin{abstract}
Resumo
Constata-se um interesse cada vez maior na utilização de ferramentas que apliquem o conceito Building Information Modeling (BIM), mas, em muitos casos, os projetistas ainda não encontram suporte tecnológico que proporcione uma transição eficiente do método de trabalho atual para um modo de trabalho que aproveite de fato as potencialidades trazidas pelo BIM. Em razão da dificuldade em encontrar referências sobre a implementação do BIM em projetos de Sistemas Prediais, em especial no Brasil, este trabalho procurou contribuir aproveitando experiências vividas do uso de tecnologias recentes e da abordagem BIM através de ferramentas atualmente disponíveis. Ao elaborar um projeto de instalações em CAD2D e CAD3D, foram observadas dificuldades no aproveitamento das informações e na correta interpretação da respectiva representação pelos profissionais envolvidos. Um modelo BIM das instalações hidráulicas do mesmo projeto foi produzido e foi feita uma comparação com as informações geradas pelo processo anterior. Como resultado, este trabalho apresenta os ganhos no que diz respeito ao conjunto de informações inseridas no projeto.
\end{abstract}

Palavras-chave: BIM. Modelagem 3D. Instalações prediais.

\begin{abstract}
There has been a growing interest in the use of tools that apply the concept Building Information Modeling (BIM), but in many cases the designers still do not find technological support to provide an efficient transition from the current working method for a job, so that in fact enjoy the potential brought by BIM. Because of the difficulty in finding references on the BIM implementation about projects of building systems, especially in Brazil, this study sought to contribute taking advantage of experiences in use of latest technology and of the BIM approach using currently available tools. In preparing a facilities design with CAD2D and CAD3D, difficulties were observed in the use of information and correct interpretation of its representation by the professionals involved. A BIM model of the hydraulic systems of the same project was produced and a comparison was made with the information generated by the previous process. As a result, this work presents gains with regard to all information inserted in the project.
\end{abstract}

Keywords: BIM. 3D model. MEP Design.

\section{INTRODUÇÃO}

De acordo com Eastman et al. (2011), o conceito de Building Information Modeling (BIM) é a integração entre o projeto e o processo de construção, resultando em mais qualidade nos edifícios e uma redução do custo e da duração do projeto. Atrelando ao projeto, além da geometria precisa, todos os dados necessários para construção, fabricação e demais necessidades que permitam obter o modelo para gerenciar o ciclo de vida da edificação.

\footnotetext{
1 BATISTA, LIDIANE M.; FERREIRA, SERGIO L. Avanços no intercâmbio de dados do projeto de instalações do CAD3D ao BIM em experiências representativas. In: ENCONTRO BRASILEIRO DE TECNOLOGIA DE INFORMAÇÃO E COMUNICAÇÃO NA CONSTRUÇÃO, 7., 2015, Recife. Anais... Porto Alegre: ANTAC, 2015.
} 
As disciplinas de projeto nem sempre caminham no mesmo ritmo com relação aos avanços da tecnologia da informação. O histórico da criação de softwares específicos, que aplicam o conceito BIM, reflete a demanda das disciplinas pelo uso de novas tecnologias. O Revit Architecture, por exemplo, teve sua primeira versão lançada em abril de 2000 (JUSTI, 2008). A Autodesk, no ano de 2002, fez a aquisição da Revit Technology Corporation e no ano de 2006 lançou uma versão do Revit MEP para instalações. Entre o período de lançamento do software de arquitetura e o de instalações, houve uma defasagem de seis anos.

Para os projetos de instalações, houve grande dificuldade em encontrar fontes de referência exemplificando tentativas ou aplicações do conceito BIM desde a concepção inicial do projeto. Dos estudos citados por Eastman et al. (2011), de dez casos analisados, apenas cinco foram listados com o uso de softwares baseados em BIM voltados para elaboração de sistemas prediais. $\mathrm{O}$ mesmo cenário se repete em publicações acadêmicas relacionadas ao tema BIM, onde grande parte aborda conceitos como gerenciamento em BIM, implementação em escritórios de projetos, onde a empresa observada é usualmente voltada para arquitetura (ENTAC, 2014). Os estudos encontrados que relatam o uso do BIM no processo de projeto e construção para instalações prediais confirmam a dificuldade encontrada para se utilizar tais ferramentas. Para Souza, Amorim e Lyrio (2009), Martins e Monteiro (2013), Czmoch e Pekala (2014), Eastman et al. (2011), uma das questões é a abordagem regional com a necessidade de adequação aos padrões e normas, assim como as questões legais na aplicação das ferramentas.

A verificação do projeto de instalações prediais é bastante complexa no método tradicional de trabalho, dificultando a procura por interferências, erros ou omissões, sendo quase impossível perceber determinadas falhas no projeto ao ser feita a compatibilização com sobreposição de camadas no desenho 2D. No momento da contratação dos serviços de instalação ou da compra de materiais é comum ainda faltarem muitas definições devido ao grande número de itens que compõem o projeto, e, muitas vezes, o problema acaba sendo resolvido durante a execução da obra (GUO; TURKAN; JAHREN, 2014).

Segundo relatado em pesquisas, a execução do projeto de sistemas prediais pode representar ou mesmo exceder cerca de $50 \%$ dos custos de projeto para edificações de alta complexidade como hospitais, laboratórios e indústrias de biotecnologia, sendo estes projetos apontados como um dos maiores desafios de coordenação (KHANZODE; FISCHER; REED, 2008) (LEE; KIM, 2014). Entretanto, apesar dos benefícios que pode representar a introdução do BIM para projetos de sistemas prediais, a adesão dos escritórios de instalações acontece de forma mais lenta que nas demais disciplinas e quando se buscam relatos de experiências, poucos escritórios oferecem este serviço para que possam relatar as dificuldades encontradas.

O objetivo desse estudo é experimentar, compreender e analisar como a introdução de um processo de projeto baseado em BIM afeta a inserção, a representação e a interpretação dos dados contidos no projeto pelos projetistas e pelos responsáveis pela execução. $O$ intercâmbio de dados é referente às informações trocadas entre os intervenientes no projeto.

\section{BIM E BENEFÍCIOS PARA INSTALAÇÕES}

Czmoch e Pekala (2014) apontam como resultado do processo em BIM, uma redução de $10 \%$ do tempo gasto no projeto e de $80 \%$ a mais de precisão em relação ao projeto elaborado pelo método tradicional. No estudo realizado por Czmoch e Pekala (2014), o modelo BIM foi utilizado durante a execução, principalmente para consultar o projeto de instalações prediais. $O$ projeto foi seguido à risca e todas as colisões haviam sido solucionadas durante a fase de projeto, não restando nenhum conflito durante a execução. 
Em outro estudo, a detecção de conflitos é relatada como um dos maiores benefícios do BIM, pois reduziu aproximadamente $20 \%$ do retrabalho e economizou entre $5 \%$ a $10 \%$ dos custos de projeto de sistemas prediais (GUO; TURKAN; JAHREN; 2014). Além disso, houve uma melhor visualização e compreensão do projeto de instalações.

O modelo BIM pode ser produzido em diferentes níveis de detalhamento (levels of detail/development LoD), sendo que para Sistemas Prediais, os modelos geralmente são elaborados no nível 400, para construção e fabricação. Farinas (2013) descreve os níveis de desenvolvimento do modelo, segundo uma classificação do American Institute of Architects (AIA):

- Level 100: representação gráfica dos elementos;

- Level 200: representação gráfica dos elementos como um sistema genérico, com informações aproximadas de tamanho, quantidade, forma, localização e orientação;

- Level 300: representação gráfica dos elementos como um sistema específico, com quantidades, tamanho, forma, localização e orientação;

- Level 400: representação gráfica dos elementos como um sistema específico, com quantidades, tamanho, forma, localização, orientação, detalhamento, fabricação, montagem e informações de instalação;

- Level 500: representação de campo exata do tamanho, forma, localização, quantidade e orientação.

\section{MODELO CAD 3D}

A Ziggurat Systems é uma empresa israelense, sediada em Nova lorque, que oferece serviços de compatibilização em 3D. Essa compatibilização é realizada através de uma ferramenta chamada B4UBTM que funciona por meio de um plug-in dentro do AutoCAD e do Navisworks. Esse plug-in permite a inserção de dados paramétricos 3D dentro do projeto em 2D. Esses dados são relacionados ao volume e posição geométrica dos componentes no modelo. Além do plug-in de modelagem, a Ziggurat Systems oferece ao cliente um programa para visualizar as análises de conflitos, ilustrado na figura 1.

Figura 1 - Visualizador Ziggurat Viewer

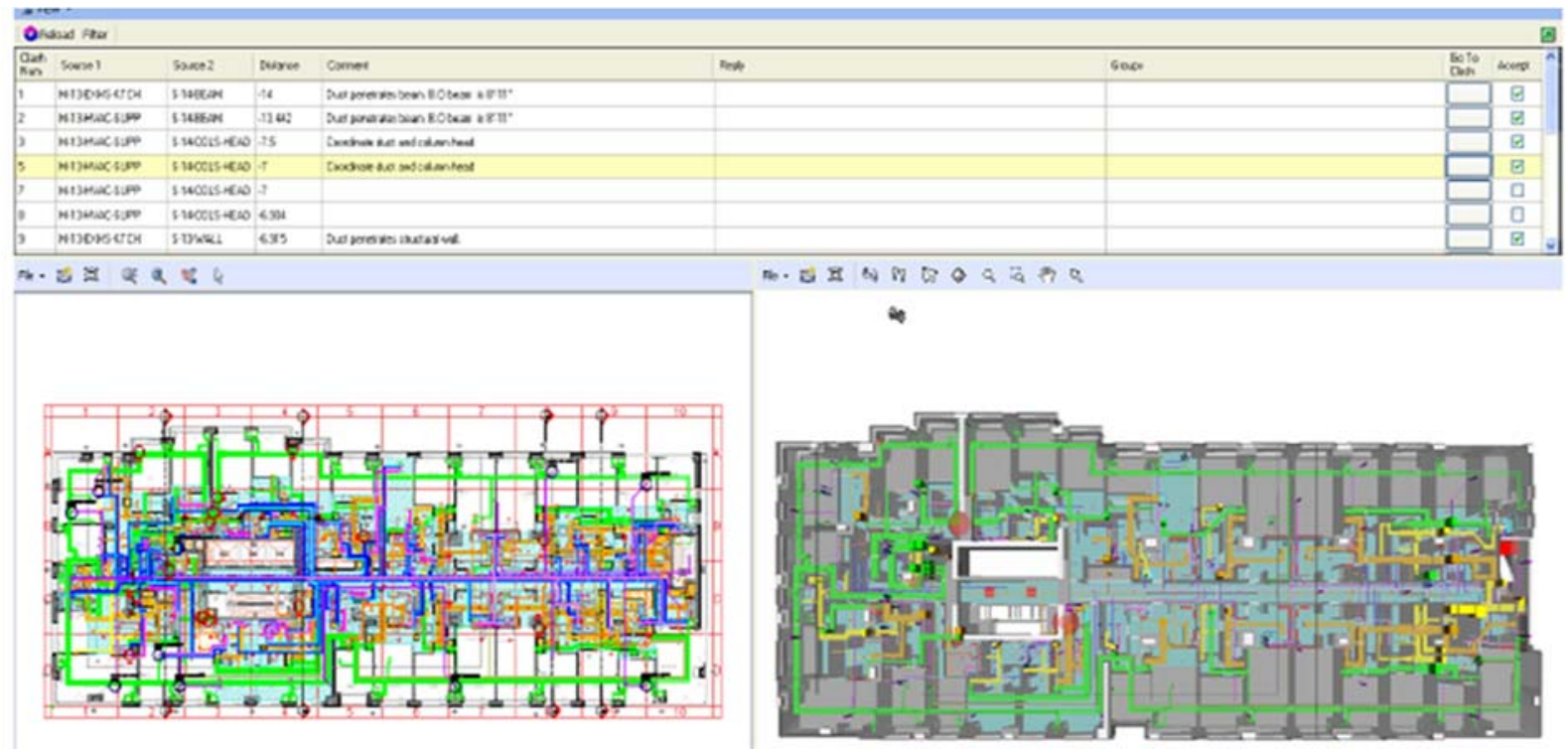

Fonte: Ziggurat, 2011. 
Este visualizador possui na parte superior uma listagem dos conflitos encontrados que pode ser exportado tanto para dentro do desenho em CAD2D e inserido na prancha a ser plotada e enviada à obra, quanto pode ser exportado para uma planilha. Na parte inferior o visualizador possui duas telas. A tela da esquerda corresponde ao projeto em CAD2D e a da direita ao modelo 3D que pode ser aberto e analisado também pelo Navisworks. Ao selecionar qualquer item da lista, o ponto correspondente no modelo é automaticamente ampliado na tela da direita. O mesmo acontece quando é selecionado um ponto da planta em 2D representada na tela esquerda, o modelo também é ampliado no local selecionado permitindo que o projetista navegue pelo modelo e verifique os conflitos encontrados. No material enviado ao cliente estão inseridos na planta 2D a marcação e identificação dos conflitos, uma tabela com a descrição dos mesmos e imagens 3D para facilitar a compreensão, conforme pode ser visto na figura 2.

Figura 2 - Conflitos representados no CAD2D

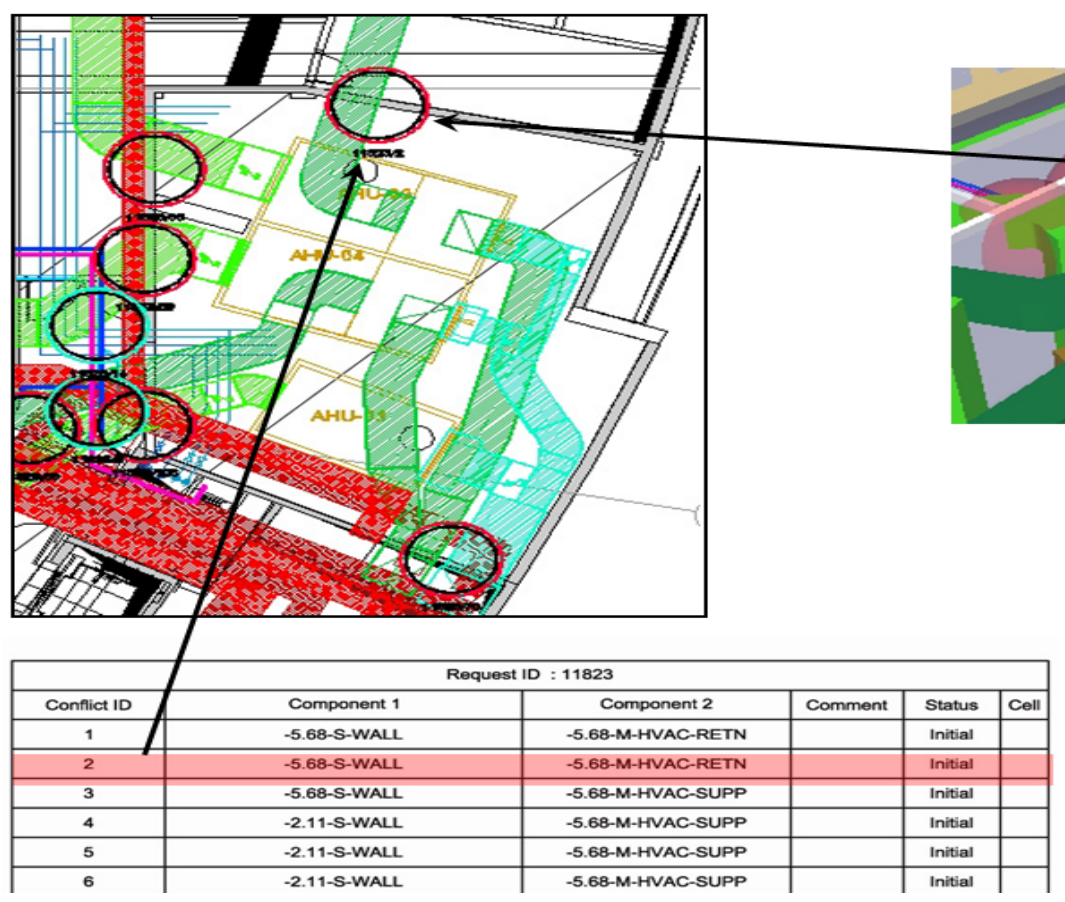

Fonte: Ziggurat, 2011.

\section{PROJETO DE INSTALAÇÕES EM CAD2D E CAD3D}

O projeto analisado faz parte do escopo de trabalho de uma empresa de projetos de instalações de pequeno porte. Resumem-se abaixo alguns dados do seu perfil:

- Número de projetistas: 41 (9 engenheiros, 14 desenhistas, 14 projetistas e 4 estagiários);

- Tempo de atuação no mercado: 27 anos;

- Porte dos projetos: variável (geralmente entre 1.000 m2 a 30.000 m2);

- Tipos de projetos: comerciais, industriais, institucionais como, por exemplo, hospitais, escolas, órgãos públicos.

O projeto de instalações prediais analisado faz parte de um processo de projeto onde estão envolvidas empresas distintas, como escritórios de arquitetura, estrutura, fundações, luminotécnico e paisagístico. O escopo de contratação do cliente solicitava que os projetos fossem desenvolvidos com o conceito BIM, mas não cobrava formatos específicos de entrega, como por exemplo .rvt, formato produzido pela plataforma Revit da Autodesk®. 
Devido às restrições do mercado de projetos, a empresa entendeu que as instalações em CAD 3D já atenderiam à necessidade e não houve concorrentes que garantissem a modelagem desde o princípio em BIM dentro do preço que o cliente estava disposto a pagar. As demais disciplinas entregaram o projeto elaborado através do Revit.

O fluxo de trabalho iniciou-se logo após a contratação da equipe de projetistas, e o contato inicial entre os responsáveis aconteceu em uma reunião onde foram definidos os conceitos iniciais e necessidades da edificação. A Arquitetura foi a primeira a iniciar os trabalhos e realizou, com as demais disciplinas, reuniões para análise do estudo preliminar e troca de informações sobre áreas para espaços técnicos, escolha da solução estrutural, cronogramas e etapas a serem cumpridas para as entregas do projeto.

Foi criado um repositório virtual para reunir os projetos, planilhas e documentos para que todos os projetistas tivessem acesso. Era necessário que os mesmos enviassem, via cadastro, as informações para este repositório virtual.

A empresa de instalações acessou e recolheu as informações disponíveis, e começou o desenvolvimento do projeto de instalações. O fluxo de trabalho dentro do escritório de instalações era dividido entre quatro equipes: elétrica, sistemas eletrônicos, ar condicionado e hidráulica. Essas equipes trabalhavam sob a coordenação geral de uma equipe de modelagem 3D. A coordenação de instalações ficou responsável por analisar as informações, repassar aos projetistas e garantir que houvesse interação entre as subdisciplinas de instalações prediais. Além dessa atividade, seria a responsável por elaborar o modelo 3D a partir da planta, com o auxílio dos projetistas responsáveis que deveriam acompanhar e esclarecer quaisquer dúvidas que surgissem do processo de modelagem.

Nas datas previstas, o cliente recebeu os desenhos em CAD 2D, planilhas, memoriais, e também o modelo 3D, que tinha como função apenas permitir a visualização geométrica das instalações no projeto e auxiliar a equipe de Arquitetura na detecção de conflitos, pois era passível de ser exportado para a plataforma Revit, uma ferramenta baseada em BIM e que foi utilizada pela arquitetura e estrutura. No cronograma do cliente, o projeto foi dividido em duas fases: anteprojeto e executivo.

\subsection{Descrição do Empreendimento}

A experiência observada é o processo de elaboração do projeto de instalações de uma edificação comercial que irá abrigar um laboratório de exames clínicos de uma rede padronizada, mas com unidades diferenciadas para atender a demanda da região em que está inserida. O prédio é caracterizado por dois blocos distintos, interligados pelo subsolo. $O$ primeiro bloco de três pavimentos que compõe a área de operação da clínica e o segundo bloco, térreo, comporta a área de apoio à funcionários, com copa, vestiários, e uma parte da área técnica.

A clínica possui um escopo específico e complexo para o projeto de instalações prediais, além da personalização dos espaços a que os projetos devem atender. Paredes de drywall, marcenarias de piso a teto, ambientes climatizados, com tratamento do ar em áreas específicas, solicitam que o projeto tenha o máximo de integração entre as disciplinas para estar adequado ao padrão exigido pelo cliente. A área total de construção do empreendimento é de 1.853,07 m2.

O serviço que o cliente desejava era a modelagem das instalações prediais em uma determinada ferramenta BIM, mas o escopo de serviço da empresa de projetos de instalações oferecia a modelagem em CAD 3D que atendia as expectativas do cliente, já que a intenção era possibilitar a compatibilização para detecção de conflitos. 

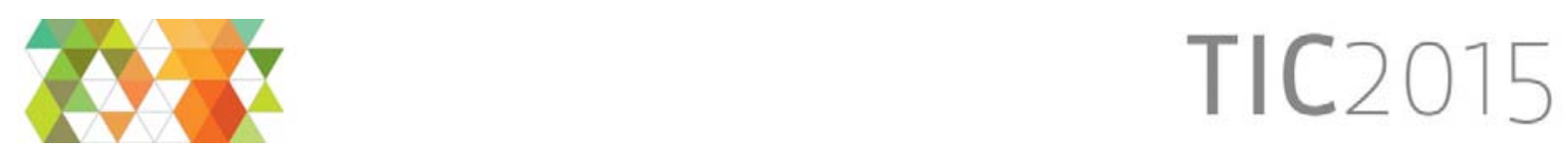

Os projetos contratados e que foram modelados em CAD 3D foram:

- Ar condicionado, ventilação e exaustão;

- Sistemas Elétricos;

- Sistemas Hidráulicos;

- Sistemas Eletrônicos.

No prazo definido pelo cronograma não foi considerado o tempo para compatibilização e integração dos projetos entre as fases de anteprojeto e executivo, o que gerou conflitos entre os prazos estipulados e a troca de informações no tempo necessário, havendo a necessidade de revisão do projeto executivo depois da data prevista para entrega final. Como não estava sendo utilizado um modelo integrado, uma empresa deveria esperar receber a informação ou revisão de outra empresa para então adequar o projeto pertinente à sua disciplina, de forma que atendesse as novas solicitações do projeto como um todo. Esta troca de informações repete o processo tradicional que se manteve nesse projeto apesar do uso do software BIM por parte das equipes de projetos.

Os projetos foram desenvolvidos no escritório de instalações da forma a seguir: os projetistas desenhavam em programas de CAD 2D, como o CADDPROJ2 e outros softwares específicos para a área de projeto em que atuam. Esses softwares possibilitam a geração de cálculos, planilhas de materiais e especificações. A partir do desenho pronto, outro profissional da empresa executa a modelagem em CAD 3D. Essa pessoa atua com uma equipe para transcrever o projeto do modelo bidimensional para o tridimensional, e durante esse processo, em constante contato com os projetistas, procura-se solucionar boa parte dos conflitos de colisão entre as instalações, além de garantir melhor compreensão do projeto entre todos os envolvidos da equipe de instalações.

Através do diretório da Ziggurat Systems, o Ziggurat Coordination Manager (ZCM), um arquivo novo de CAD é criado e este arquivo, quando aberto, permite importar os dados do arquivo de instalações em CAD2D, podendo assim iniciar a modelagem com a inserção dos dados 3D. Os dados importados devem ser selecionados por tipo de disciplina e subsistema, por exemplo, disciplina de elétrica, subsistema de iluminação. É possível ainda selecionar uma terceira categoria como, por exemplo, inserir a informação se é luminária de piso. Dessa forma o software separa os dados importados por camadas (layers) e disciplinas, possibilitando utilizar a ferramenta de filtros posteriormente, além de conseguir identificar os conflitos no diretório do ZCM como também no programa Navisworks.

Após a importação dos projetos de instalações para o arquivo de coordenação, inicia-se o processo de modelagem 3D, com a inserção dos parâmetros. Para uma tubulação é necessário, por exemplo, inserir no mínimo dois parâmetros, podendo ser o nível da tubulação (topo, eixo ou face inferior) e diâmetro, devendo ser informada pelo menos uma posição de nível. Dessa forma, o programa consegue gerar o modelo. Caso os dados sejam inseridos incorretamente, o programa não detecta falhas na inserção, sendo fundamental a análise do responsável. Após a finalização da inserção dos dados no arquivo de coordenação CAD é selecionada uma ferramenta para geração do modelo 3D. Este modelo pode ser gerado diretamente no CAD, ou podemos enviar o modelo para o diretório Ziggurat Coordination Manager, e este diretório permite, ao selecionar o modelo, solicitar a geração de uma análise do mesmo. Essa análise pode ser exportada e aberta no Navisworks e no AutoCAD, ou se pode abrir e verificar a análise pelo visualizador da Ziggurat. $O$ modelo exportado para o CAD pode ser aberto no Navisworks. O modelo 3D no formato dwg foi o escolhido para ser enviado para as demais disciplinas de arquitetura e estrutura inserirem

\footnotetext{
${ }^{2}$ Softwa re disponível no site: বhttp://highlightnews.com.br/Caddproj/Caddproj_Saiba_Mais.aspx> acesso em maio de 2015.
} 
no modelo Revit.

Os principais problemas detectados na elaboração do modelo 3D, por uma equipe que não era a que projetou originalmente as instalações, foram relacionados às dificuldades de interpretação, assim como soluções apresentadas no projeto 2D que quando transcritas para 0 3D não funcionavam, principalmente em relação aos desvios de dutos de climatização. O modelo em 3D estava baseado nas informações das plantas baixas e dos cortes desenvolvidos pelos projetistas que nem sempre mostravam os trechos mais críticos. Ficando a cargo do responsável pelo 3D muitas das soluções de posicionamento e desvios de trechos de tubulações, dutos e eletrocalhas. A tabela 1 mostra os resultados em relação ao tempo total gasto no projeto.

Tabela 1 - Horas de projeto das instalações prediais

\begin{tabular}{lrrrr}
\hline Setor & Anteprojeto & Projeto Executivo & Revisão & Total \\
\hline Modelagem 3D - instalações & 64 & 99 & 11 & 174 \\
\hline Ar Condicionado & 124 & 128 & 3 & 255 \\
\hline Elétrica & 112 & 99 & 0 & 211 \\
\hline Hidráulica & 76 & 105 & 4 & 185 \\
\hline Sistemas & 57 & 88 & 0 & 145 \\
\hline Total & $\mathbf{4 3 3}$ & $\mathbf{5 1 9}$ & $\mathbf{1 8}$ & $\mathbf{9 7 0}$ \\
\hline
\end{tabular}

Fonte: Elaborado pelos autores.

Entre os problemas detectados pela modelagem 3D, as interferências existentes entre as instalações no entreforro, como desníveis do forro de gesso, caixa das luminárias, sancas de iluminação, colidindo com grelhas, difusores, equipamentos de climatização, eletrocalhas e tubulações, puderam ser observados no modelo e apontados para correções nos projetos. Soluções que tinham sido apontadas nas plantas e cortes em 2D quando modelados em 3D não eram possíveis de serem executadas. A imagem apresentada na figura 3 representa 0 corte 2D da casa de máquinas de ar condicionado, nela é possível observar que os dutos próximos da laje do teto estão alinhados, quando na verdade esse posicionamento é impossível de seguir durante a execução, pois causaria colisões entre eles. A modelagem 3D permitiu que os desvios necessários fossem previstos, deslocando os dutos do posicionamento inicial ao se observar as colisões existentes (figura 4).

Figura 3 - Casa de Maquinas Ar Condicionado - Corte em CAD 2D

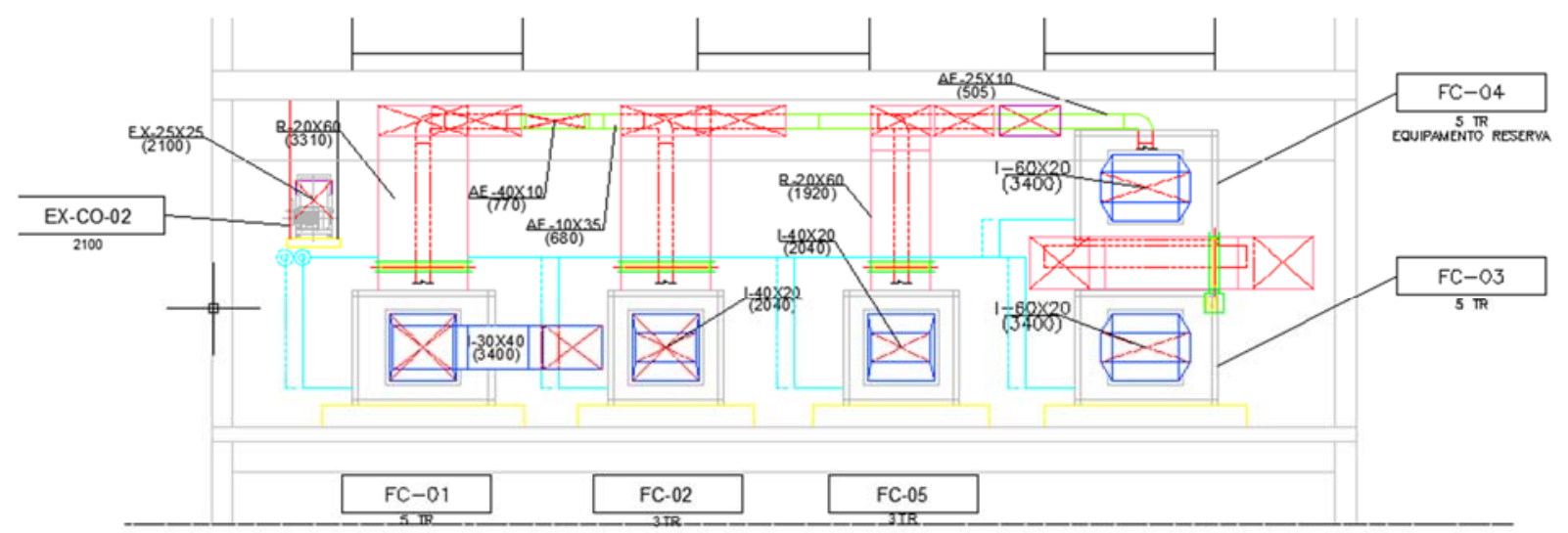

CORTE BB

Fonte: Elaborado pelos autores. 
Figura 4 - Casa de Maquinas Ar Condicionado - Modelo CAD 3D

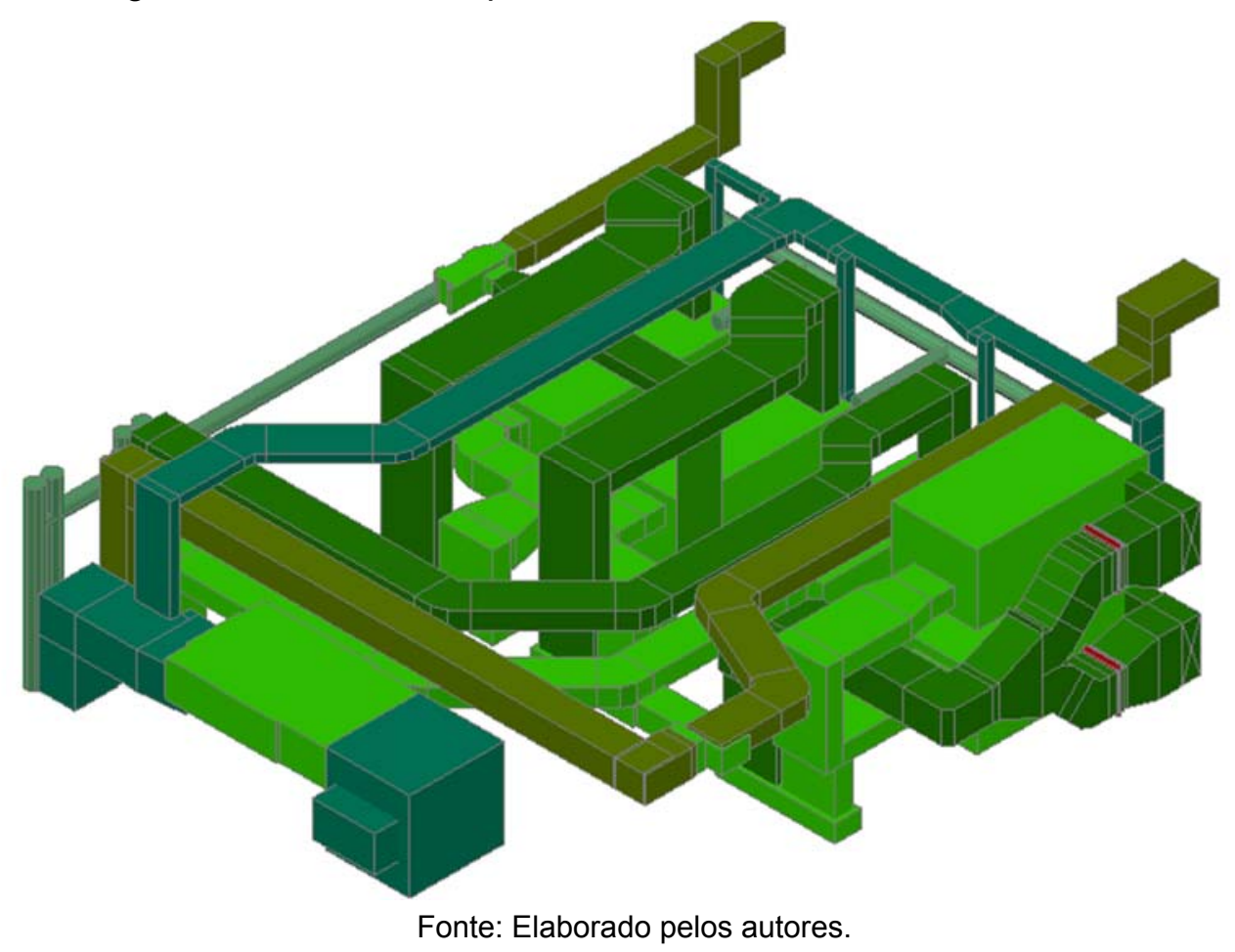

As entregas do projeto foram realizadas de forma que a disciplina de arquitetura recebesse o anteprojeto de instalações composto pelas plantas baixas em CAD 2D para análise dos pontos de instalações, alterações de layout. Este anteprojeto foi entregue uma semana antes do modelo CAD 3D. Este intervalo possibilitou fazer a compatibilização entre as instalações, além de receber os demais comentários da equipe de arquitetura. Na fase do executivo não foi estipulado um prazo de compatibilização final entre os projetos, causando um atraso de dez dias entre a entrega final prevista e a realizada. Os projetos foram iniciados no dia 06 de outubro e finalizados no dia 12 de dezembro, num total de 67 dias. Todas as adequações após essa entrega foram em relação à dúvidas técnicas ou alterações, por exemplo, de conceitos, não sendo geradas por incompatibilidades encontradas no projeto.

\section{INTRODUÇÃO DA MODELAGEM EM BIM NO PROJETO DE INSTALAÇÕES}

Com a intenção de verificar a qualidade das informações contidas no projeto, foi realizada uma tentativa de introdução do modelo BIM em substituição ao modelo CAD 3D. Foi utilizado o mesmo material recebido para o modelo CAD 3D: plantas baixas e cortes do projeto de instalações. Foram escolhidos os softwares Revit e DDSCAD, mas diversos foram os problemas enfrentados para a realização dos modelos, sendo possível finalizar apenas a modelagem das instalações hidráulicas de água fria no Revit. Apesar das dificuldades, houve uma análise dos recursos disponíveis em ambos os softwares.

Primeiramente foram pesquisados projetistas que tinham conhecimento prévio nas ferramentas, mas não houve sucesso, pois os mesmos fizeram projetos pilotos acompanhados do suporte de treinamento na época de aquisição do software pela empresa na qual trabalhavam, não acumulando nenhuma experiência após o treinamento que permitisse desenvolverem o projeto sem essa assistência. Foi então realizado pelos autores uma tentativa de modelagem no software DDSCAD utilizando o arquivo de arquitetura proveniente do Revit como base. Ao exportar esse arquivo no formato Industry Foundation 
Classes (IFC), o layout interno da arquitetura, as paredes em drywall, os mobiliários planejados, as louças e outros equipamentos não vieram para o arquivo do DDSCAD. Foi identificado, portanto, uma falha na importação ou exportação do IFC proveniente do Revit, além de outros erros de importação que não foram possíveis solucionar no prazo adequado.

O modelo 3D no DDSCAD foi descartado, pois não foi encontrada uma solução para os problemas de importação do IFC, sendo introduzida uma nova tentativa no modelo Revit. Para a modelagem no Revit foi utilizado o aplicativo TigreCAD® desenvolvido pela Ofcdesk para a Tigre S/A Tubos e Conexões. Este aplicativo oferece ferramentas especiais para possibilitar o desenvolvimento de projetos de instalações hidráulicas. Mesmo com o auxílio do aplicativo, houve a necessidade de reinstalar os programas em razão dos erros frequentes que ocorriam durante a modelagem. Para realizar a modelagem de apenas um pavimento e um único sistema, no caso água fria, o tempo gasto foi superior ao tempo gasto na modelagem no CAD 3D. No entanto, o resultado parcial obtido contêm mais informações e uma maior facilidade de interpretação, conforme pode ser observado na figura 5.

No processo de elaboração dos modelos, foi priorizado o modelo hidráulico pela maior facilidade para os projetistas envolvidos compreenderem e a analisarem as ferramentas, pois não possuíam a experiência com a modelagem em BIM. $\mathrm{Na}$ análise das funcionalidades disponíveis pelas ferramentas, cabe destacar as diferenças entre o processo tradicional e as possibilidades advindas do BIM. Ferramentas de cálculo da rede para dimensionamento da tubulação, listas de materiais, entre outras também são disponibilizados pelo software DDSCAD.

A tabela 2 resume as funcionalidades que o modelo BIM proporciona ao projetista de instalações hidráulicas, vinculando os cálculos ao modelo. No processo CAD 2D tradicional, os cálculos são elaborados em planilhas separadas do desenho, podendo ocorrer, sem que seja notado, uma divergência entre a informação indicada no desenho e a lista de material. No software Revit, foi adicionado uma ferramenta disponibilizada pela TigreCAD $®$, as funcionalidades principais dessa ferramenta só estão disponíveis na licença paga. Essa ferramenta permite elaborar com mais facilidade o projeto hidráulico no Revit. $\mathrm{O}$ cálculo do reservatório disponibilizado pela ferramenta tem três opções de sistema de distribuição, mas não contempla rede pressurizada com reservatório inferior. Depois de inseridos os pontos de consumo e de toda a rede traçada, é possível checar todo o projeto com a utilização dos cálculos da Bomba e da tubulação (figura 6), assim como obter a listagem de todos os materiais utilizados no desenho.

Tabela 2 - Processo de cálculo do projeto hidráulico

\begin{tabular}{lll}
\hline Atividade & \multicolumn{1}{c}{ CAD 2D } & \multicolumn{1}{c}{ BIM } \\
\hline $\begin{array}{l}\text { Cálculo } \\
\text { Reservatório }\end{array}$ & $\begin{array}{l}\text { Calculo inicial, elaborado com o uso de } \\
\text { planilhas, volume baseado na população. }\end{array}$ & $\begin{array}{l}\text { Revit: Cálculo inicial do volume baseado na população } \\
\text { (Norma). }\end{array}$ \\
\hline $\begin{array}{l}\text { Dimensionamento } \\
\text { tubos }\end{array}$ & $\begin{array}{l}\text { Elaborado com uso de planilhas, cálculo } \\
\text { baseado no número de pontos alimentados. }\end{array}$ & $\begin{array}{l}\text { Revit: Elaborado automaticamente depois de desenhado } \\
\text { todos os sistemas e conexões, seleção manual dos tubos } \\
\text { e componentes do sistema. }\end{array}$ \\
\hline Vazão da rede & $\begin{array}{l}\text { Elaborado com uso de planilhas, baseado no } \\
\text { diametro dos tubos. }\end{array}$ & Idem dimesionamento de tubos \\
\hline Pressão da Rede & $\begin{array}{l}\text { Elaborado com uso de planilhas, baseado na } \\
\text { vazão, comprimento, desnível e perda de } \\
\text { carga nas conexões. }\end{array}$ & Idem dimesionamento de tubos \\
\hline Bombas & $\begin{array}{l}\text { Elaborado com uso de planilhas, Baseado na } \\
\text { vazão e pressão da rede. }\end{array}$ & Revit: Elaborado automaticamente. \\
\hline Perda de carga & $\begin{array}{l}\text { Elaborado com uso de planilhas. Baseado nas } \\
\text { conexões e desníveis da rede. }\end{array}$ & Idem dimesionamento de tubos \\
\hline Lista de Materiais & $\begin{array}{l}\text { Elaborado com uso de planilhas. Baseado no } \\
\text { levantamento manual dos dados do desenho. }\end{array}$ & Revit: Elaborado automaticamente. \\
\hline
\end{tabular}


Figura 5 - Modelagem sistema de água fria no Revit

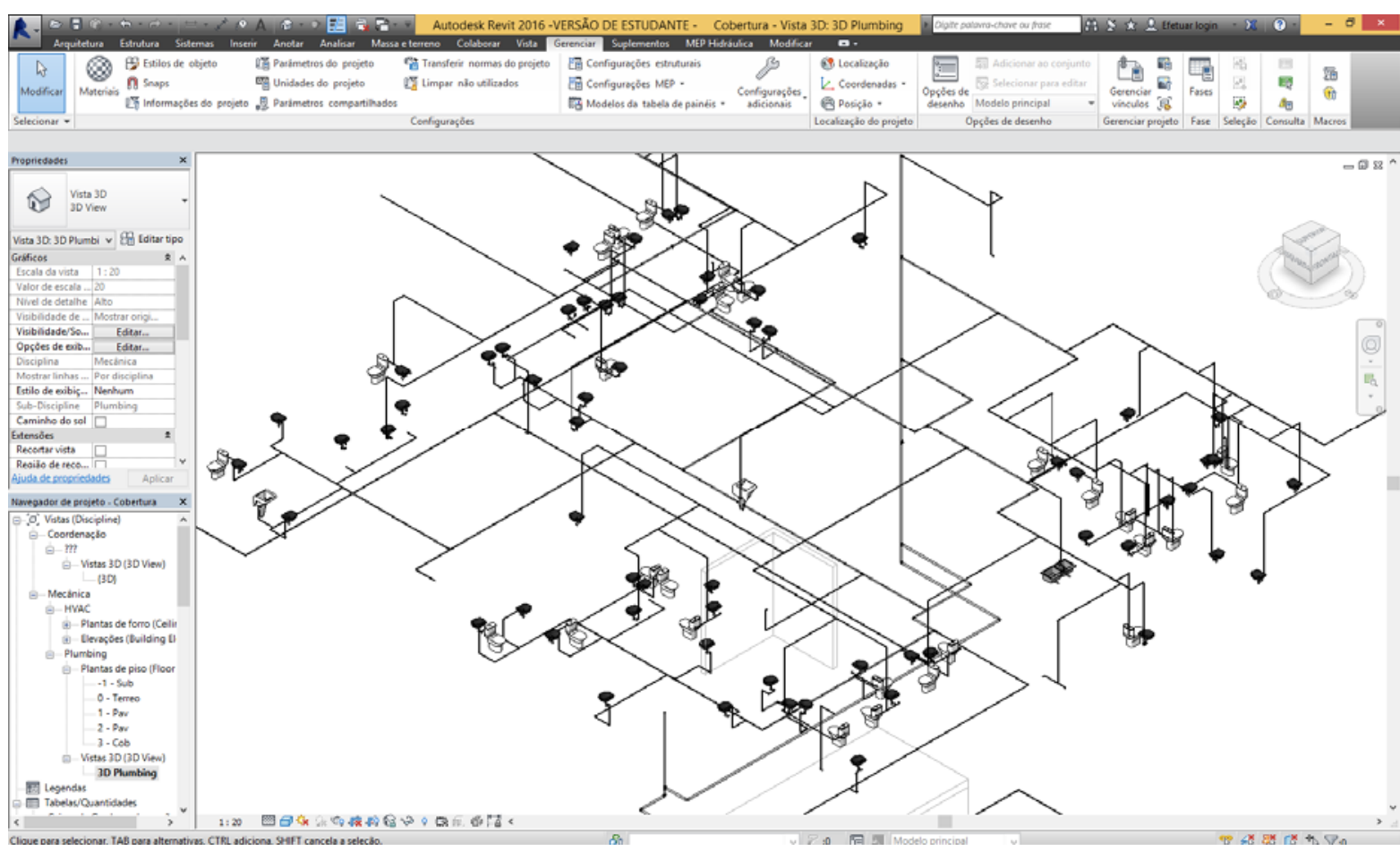

Fonte: Elaborado pelos autores.

Figura 6 - Ofcdesk TigreCAD® - Ferramenta de cálculo do sistema

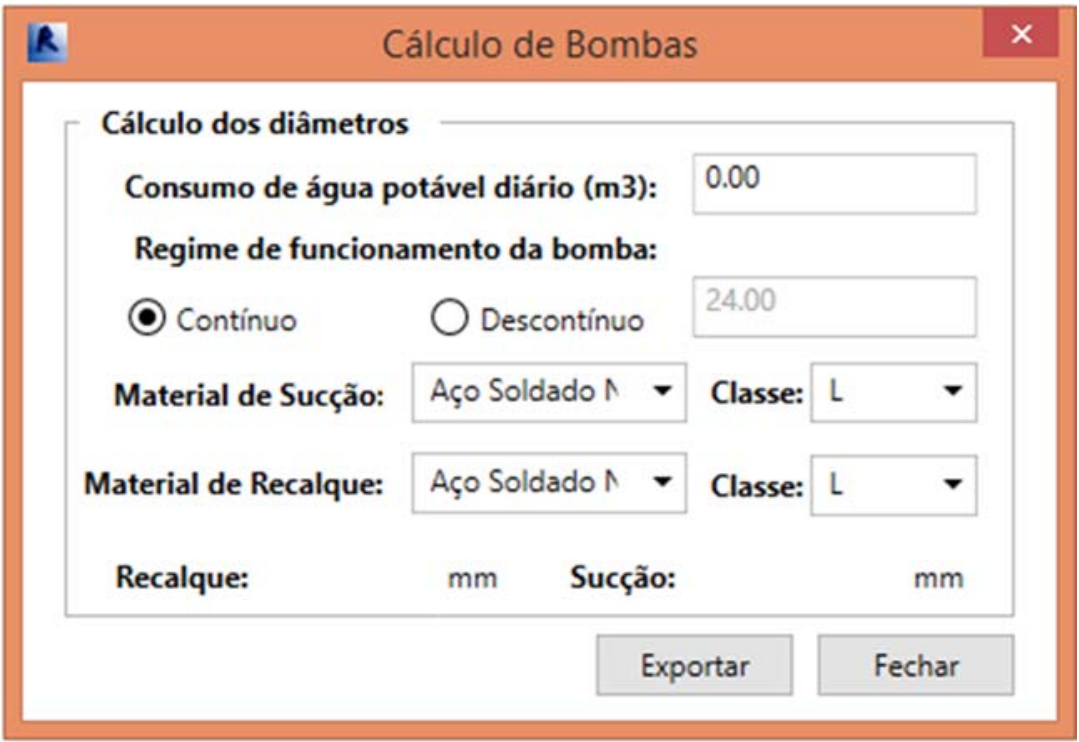

Fonte: Elaborado pelos autores.

\section{CONCLUSÕES}

Neste estudo, ao realizar o modelo BIM das instalações hidráulicas em comparação ao CAD2D/3D, foi possível verificar as diferenças no processo de inserção das informações no projeto. Enquanto no BIM as informações vão sendo incorporadas ao modelo no decorrer da elaboração do projeto, no processo CAD2D/3D as informações ficam separadas, dificultando sua interpretação e atualização. A possibilidade de desvio e perda dessas informações facilita o erro no trabalho dos profissionais envolvidos no processo CAD2D. Muitas vezes, 
para evitar erros, uma estratégia comumente utilizada é deixar muitas das informações em aberto ou generalizadas para serem resolvidas durante a execução da obra. Esse artifício não pode ser utilizado no modelo BIM, onde são necessárias que muito mais informações sejam inseridas desde o princípio, gerando um projeto mais fidedigno à futura edificação. Ou seja, o BIM seria um modelo do edifício virtual completo, facilitando o trabalho dos profissionais ao inserir todos os itens de maneira detalhada, permitindo um levantamento de dados precisos, além da detecção de interferências ou identificação da ausência de trechos e componentes do projeto.

Assim, as ferramentas disponíveis para elaboração de projetos de instalações prediais em BIM trazem muitos benefícios para o processo de projeto e para a qualidade final do produto entregue ao cliente. Problemas recorrentes tais como informações incompletas, redes desconectadas entre pavimentos diferentes, vazão e pressão da rede calculada erroneamente, podem ser facilmente identificados no modelo virtual da construção. No processo $2 \mathrm{D}$ tradicional, os cálculos são feitos à mão ou em planilhas para então serem inseridas as informações no desenho do projeto. No ambiente BIM, as informações de base dos cálculos do projeto estão incorporadas ao modelo, estando disponíveis para conferência e correção a qualquer momento. Sendo assim, desenhos e cálculos do sistema permanecem integrados e quaisquer atualizações das características da rede são propagadas e se transformam em alterações na memória de cálculo dos sistemas e nos quantitativos de materiais. Essa integração entre o modelo e os documentos do projeto facilita o trabalho no canteiro de obras, garantindo maior fidelidade ao que foi proposto e isso evita erros por omissão de informação. Os benefícios da análise de conflitos entre as disciplinas já eram possíveis com o CAD 3D. O grande ganho no BIM verificado nesse estudo foi a integração de todas as especificações, possibilitando o acesso sempre atualizado às informações do projeto.

Entretanto, os projetos de instalações prediais precisam de melhorias no processo. Além das dificuldades enfrentadas para utilizar as ferramentas BIM, o que se vê de mais deficiente é a falta de capacitação das equipes de projetistas, como também das empresas de projetos. Os poucos projetistas encontrados que tinham recebido algum treinamento em softwares baseados em BIM não estavam em um ambiente propício para desenvolverem a prática necessária para superar os desafios da implantação. Neste cenário, a capacitação profissional é um fator importante para a popularização do BIM, pois o uso intenso dos softwares possibilita a análise de suas funcionalidades e a adaptação dos mesmos ao mercado.

\section{AGRADECIMENTOS}

Os autores agradecem a colaboração do projetista Giovani Sacrini que auxiliou no modelo BIM, à empresa MBM Engenharia que forneceu os projetos de instalações, ao Filipe Ferreira pela disponibilização de uma licença do DDSCAD para este trabalho.

\section{REFERÊNCIAS}

CZMOCH, Ireneusz; PEKALA, Adam. Traditional Design versus BIM Based Design. Procedia Engineering, [s.I.], n. 91, p.210-215, 2014. Disponível em: <www.sciencedirect.com>. Acesso em: 27 fev. 2015.

EASTMAN, Chales et al. BIM Handbook: A guide to building information modeling for owners, managers, designers, engineers, and contractors. Hoboken: John Wiley \& Sons, Inc., 2011. 648 p. $2^{\mathrm{a}}$ ed.

ENCONTRO NACIONAL DE TECNOLOGIA DO AMBIENTE CONSTRUÍDO, 15, 2014, Maceió. Avanços no desempenho das construções - pesquisa, inovação e capacitação profissional. 
Maceió: Associação Nacional de Tecnologia do Ambiente Construído, 2014. 3502 p. Disponível em: <http://www.ufal.edu.br/eventos/entac2014>. Acesso em: 06 jan. 2015.

FARINAS, Humberto. Desenvolvimento de objetos BIM e os desafios. In: ENCONTRO NACIONAL DE EMPRESAS PROJETISTAS E CONSULTORES DA ABRAVA, 13., 2013, São Paulo.

Apresentação. São Paulo: Abrava, 2013. p. 0 - 48. Disponível em:

<http://www.abrava.com.br/down/Humberto Farina.pdf>. Acesso em: 06 jun. 2015.

GUO, Fangyu; TURKAN, Yelda; JAHREN, Charles T.. Case studies of BIM practices within mechanical contractors. In: CONSTRUCTION RESEARCH CONGRESS, 28., 2014, Atlanta. Proceedings... Atlanta: Asce, 2014. p. 1 - 10. Disponível em:

<http://dx.doi.org/10.1061/9780784413517>. Acesso em: 29 maio 2015.

JUSTI, Alexander Rodrigues. Implantação da Plataforma Revit nos Escritórios Brasileiros: relato de uma experiência.Gestão \& Tecnologia de Projetos, São Paulo, v. 3, n. 1, p.140-152, maio 2008. Mensal. Disponível em: <http://www.revistas.usp.br/gestaodeprojetos/issue/view/4231/showToc>. Acesso em: 18 jun. 2014.

KHANZODE, A; FISCHER, M; REED, D. Benefits and lessons learned of implementing building virtual design and construction (VDC) technologies for coordination of mechanical, electrical, and plumbing (MEP) systems on a large healthcare project, ITcon, Stanford, v. 13, n.8, p.324-342, junho 2008. Edição Especial: Case studies of BIM. Disponível em: <http://www.itcon.org/2008/22>. Acesso em: 10 abr. 2014.

LEE, Ghang; KIM, Jonghonn Walter. Parallel vs. Sequential Cascading MEP Coordination Strategies: A Pharmaceutical Building Case Study.Automation In Construction, [s.I.], n. 43, p.170179, 2014. Disponível em: <http://www.sciencedirect.com/science/article/pii/S0926580514000569>. Acesso em: $11 \mathrm{fev} .2015$.

MARTINS, João Poças; MONTEIRO, André. LicA: A BIM based automated code-cheking application for water distribution systems.Automation In Construction, [s.I.], n. 29, p.12-23, 2013. Disponível em: <http://www.sciencedirect.com/science/article/pii/S0926580512001495>. Acesso em: 11 fev. 2015.

SOUZA, Livia L. Alves de; AMORIM, Sergio R. Leusin; LYRIO, Arnaldo de Magalhaes. Impactos do uso do BIM em escritórios de arquitetura: oportunidades no mercado imobiliário.Gestão \& Tecnologia de Projetos,São Paulo, v. 4, n. 2, p.26-53, nov. 2009. Disponível em:

<http://www.revistas.usp.br/gestaodeprojetos/article/view/50958>. Acesso em: 16 out. 2014. 